Draft VERsion SEPTEMber 3, 2021

Typeset using LATEX default style in AASTeX61

\title{
THEORY AND SIMULATION OF SPECTRAL LINE BROADENING BY EXOPLANETARY ATMOSPHERIC HAZE
}

\author{
Z. Felfli,${ }^{1,2}$ T. Karman,${ }^{3}$ V. Kharchenko,${ }^{1,4}$ D. Vrinceanu,${ }^{5}$ J. F. Babb,${ }^{1}$ and H. R. Sadeghpour ${ }^{1}$
}

\footnotetext{
${ }^{1}$ ITAMP, Harvard-Smithsonian Center for Astrophysics, Cambridge, MA 02138

${ }^{2}$ Department of Physics, Clark Atlanta University, Atlanta, GA 30314

${ }^{3}$ Joint Quantum Centre, Durham University, Durham, DH1 3LE, United Kingdom

${ }^{4}$ Department of Physics, UConn, Storrs, CT 06269

${ }^{5}$ Department of Physics, Texas Southern University, Houston, TX 77004
}

Atmospheric haze is the leading candidate for the flattening of expolanetary spectra, as it's also an important source of opacity in the atmospheres of solar system planets, satellites, and comets. Exoplanetary transmission spectra, which carry information about how the planetary atmospheres become opaque to stellar light in transit, show broad featureless absorption in the region of wavelengths corresponding to spectral lines of sodium, potassium and water. We develop a detailed atomistic model, describing interactions of atomic or molecular radiators with dust and atmospheric haze particulates. This model incorporates a realistic structure of haze particulates from small nano-size seed particles up to sub-micron irregularly shaped aggregates, accounting for both pairwise collisions between the radiator and haze perturbers, and quasi-static mean field shift of levels in haze environments. This formalism can explain large flattening of absorption and emission spectra in haze atmospheres and shows how the radiator - haze particle interaction affects the absorption spectral shape in the wings of spectral lines and near their centers. The theory can account for nearly all realistic structure, size and chemical composition of haze particulates and predict their influence on absorption and emission spectra in hazy environments. We illustrate the utility of the method by computing shift and broadening of the emission spectra of the sodium D line in an argon haze. The simplicity, elegance and generality of the proposed model should make it amenable to a broad community of users in astrophysics and chemistry.

Keywords: exoplanetary atmosphere — haze — pseudopotential — spectral shift and broadening 


\section{INTRODUCTION}

Extrasolar planets (exoplanets) are being discovered at a breakneck pace, supplanting and transforming our understanding of planetary formation and habitability. Thousands of exoplanets have been identified using a host of detection techniques, through measurements of radial velocities, transits, and lensing (ExoOrg 2018; ExoEU 2018). Many of these observed planets harbor atmospheres, and will be amenable to further observations with the launch of next generation telescopes, including the James Webb Space Telescope, the Transiting Exoplanet Survey Satellite, and with future terrestrial telescopes, including the Giant Magellan Telescope. An Analysis of spectral compositions and parameters of exoplanetary atmospheres is a topic of high interest, but prominent spectral features in observed transmission spectra of such exoplanets are often obscured.

Atmospheric haze is the leading candidate for the flattening of spectral transmission of expolanetary occultation, a fact that is supported by observations of solar system planets, including Earth, and cometary atmospheres (Zhang et al. 2017; Deming \& Seager 2017; Seager \& Deming 2010). Radiation spectra transmitted through hazy atmospheres carry information about how these atmospheres become opaque to stellar light in transit (Pont et al. 2008; Deming \& Seager 2017; Spake et al. 2018). Recent laboratory experiments simulating hazy environments for super-Earths and mini-Neptunes atmospheres suggest that some of these atmospheres contain thick photochemically generated hazes (Hörst et al. 2018). Because hazy environments can reflect and absorb light, their conditions ought to be explored for direct imaging of exoplanets (Morley et al. 2015).

The stellar flux occultation during a planet's transit can also give clues to the persistence of an atmosphere; the wavelength dependent measurement of the planet transit depths reveal information on atmospheric atomic and molecular composition and transparency of the atmosphere. The Na I D and K I first resonance lines were modeled in absorption to constrain line-of-sight atmosphere barometric parameters and cloud depths (Seager \& Sasselov 2000). Predictions made by (Seager \& Sasselov 2000) were based on the assumption that these exoplanetary atmospheres are similar to brown and cool L dwarfs with similar effective temperatures. Water is expected to be the most spectroscopically active gas, but Na I, K I, metastable He I and CO have been identified in hot Jupiter atmospheres, and in particular in HD 209458b and HD 189733b (Seager \& Deming 2010; Sing et al. 2011; Deming \& Seager 2017; Spake et al. 2018; Pont et al. 2008, 2013). Many of the detected lines are model dependent, but Na I (and K I) resonant doublets are not, as there are no other absorbers at such wavelengths.

In methane dwarfs (Burrows et al. 2000, 2001), the K I doublet $4^{2} S-4^{2} P$ absorption line at $769 \mathrm{~nm}$ is broad and is responsible for large continuum depression, red shifted in optical spectra. The continuum broadening is induced due to collisional interaction (pressure broadening) between the radiator and background gas atoms and molecules. This lower than expected contrast of the strength of the alkali metal lines (Fortney et al. 2003) has been puzzling: subsolar elemental abundances, stellar radiation ionization, and atmospheric haze particulates have been invoked as sources for the diminished strength of the lines.

The haze hypothesis has gained currency (Burrows 2014; Deming \& Seager 2017). Spectra of radiation observed from different planetary and exoplanetary atmospheres, comets, and natural satellites reveal unusual lack of sharp spectral features, attributed to the presence of atmospheric dust, ice and haze particles (Wong et al. 2003; Greenberg \& Li 1999; West 1991; Ortiz et al. 1996; Rannou et al. 2002; West 1991; Tomasko et al. 1986). Perhaps the most compelling evidence for the direct role played by haze layers in flattening of the exoplanetary lines is the transit spectra in near-UV and mid-IR by Pont et al. (2013, 2008) of HD189733b. Lack of features in the range from 550 to $1050 \mathrm{~nm}$ in the spectra of HD 189733b is suggestive of absorption by condensates high in the atmosphere (Pont et al. 2008); a strong indication of presence of sub-micron haze clusters. In general, hazes are clouds of small (sub-micron) particulates which can produce featureless continuum opacity to light. The red hue of Jupiter is likely produced by unidentified trace species (or haze) (Burrows 2014). The transit spectra for the mini-Neptune GJ 1214b have been shown by (Kreidberg et al. 2014) to be 5-10 times flatter than a water-rich, $\mathrm{H}_{2}$-dominated atmosphere with, heralding the presence of a thick haze layer. In situ measurements of the effective density of aerosol materials in analogue Titan atmospheres confirm densities of haze-particle materials around $0.4-1.13 \mathrm{~g} / \mathrm{cm}^{3}$, which for a methane dominated hazy environment, translates to the atom/molecule number densities of a few times $10^{22} \mathrm{~cm}^{-3}$ (Hörst \& Tolbert 2013).

We present a theoretical framework for a quantum mechanical description of the shift and broadening of atomic and molecular spectral lines by dust and haze particles. Our model can be applied to shift and broadening analysis of different lines of atomic and molecular spectra, when haze particulates may have liquid or solid structures or consist of high porosity materials. Spectral calculations are obtained through the introduction of the pseudo-potential for the interaction between radiator electrons and atoms and molecules of haze particles (Szasz 1985). The pseudo-potential 
method allows us to carry out calculations for different chemical compositions of haze (Jacquet et al. 2011; Boatz \& Mario 1994). The binary collision between the radiator and haze particulate, affecting the radiator line center, is also considered (Allard \& Kielkopf 1982).

\section{METHODOLOGY}

\subsection{Modeling of Emission and Absorption Spectra in Haze Environments}

The physical nature, composition and structure of these particles is roughly known for the Earth, some solar system planets, satellites, and comets (Greenberg \& Li 1999; West 1991; Seignovert et al. 2017), but for exoplanetary atmospheres the nature of the particles is not known (Tinetti et al. 2007). Analysis and interpretation of atomic and molecular spectra observed from dusty environments is a formidable task because of complicated quantum mechanical interaction between radiating atoms/molecules and haze particles.

Another fundamental obstacle in modeling optical properties of mesoscopic dust, ice and haze particles is the stochastic nature of the haze particle distribution (Greenberg \& Li 1999; Sciamma-O'Brien et al. 2017). The simplest approaches to modeling distributions employ empirically assigned refractive indices in the classical Mie model for scattering and absorption of radiation by spherical haze particles (Lenoble et al. 2013; Seignovert et al. 2017). This model fails to describe realistic changes in atomic and molecular emission/absorption spectra because optical properties of nano-size particles cannot be described by macroscopic parameters such as the refractive index.

\subsection{Quantum Mechanical Model of Interaction Between Radiator and Haze Particles}

The electronic Hamiltonian $H_{e l}$ for the radiator-haze system can be written as:

$$
H_{e l}=H_{r}+H_{h p}+V_{r-h p},
$$

where $H_{r}$ is an electronic Hamiltonian of radiating atom or molecule; $H_{h p}$ the haze particle Hamiltonian, and $V_{r-h p}$ the potential of interaction between the haze and radiator electrons. The haze particle Hamiltonian $\hat{H}_{h p}$ includes kinetic energies and interaction potential energies of all atomic and molecular constituents.

The term $V_{r-h p}$ is given by the sum of interaction potential energies $V_{i}$ between radiator electrons and an atomic or molecular constituent in the haze at location $\mathbf{R}_{\mathbf{i}}: V_{r-h p}=\sum_{i=1}^{N} V_{i}\left(\mathbf{r}_{\mathbf{e}}, \mathbf{R}_{\mathbf{r}}, \mathbf{R}_{\mathbf{i}}\right)$, where $N$ is the total number of haze atoms and/or molecules, $\mathbf{r}_{\mathbf{e}}$ is the electron coordinate from the radiator center $\mathbf{R}_{\mathbf{r}}$. Figure 1 illustrates the basic configuration of the haze-radiator system.

There are only dozens of atoms or molecules in nano-size seed particles and millions in sub-micron haze aggregates. The set of location vectors $\left\{\mathbf{R}_{\mathbf{i}}\right\}$ determines the geometrical structure of haze, its physical properties and characteristics of interaction with radiator. For mesoscopic size "haze-radiator" system, the set $\left\{\mathbf{R}_{\mathbf{i}}\right\}$ has typically an irregular structure and resembles an amorphous or liquid cluster. Nevertheless, seed particles could be represented by nanocrystals or amorphous $\mathrm{Si}$ or $\mathrm{C}$ materials covered by polycrystalline structures of $\mathrm{H}_{2} \mathrm{O}$ and $\mathrm{CO}_{2}$ ices or $\mathrm{CH}_{4}$ droplets expected as outer shells in highly porous sub-micron haze aggregates.

\subsubsection{Mean-field spectral line shift in haze environment}

The atmospheric distribution of haze particles is non-uniform as is the distribution of atoms and molecules inside haze droplets. Our modeling of spectral line shift and broadening is focused on the quantum mechanical description of the interactions between the haze particles and the atomic or molecular radiators. The many-body interaction between the emitter and haze constituents is considered in the mean-field approximation.

The Fermi pseudopotential method (Fermi 1934) was first developed to describe species-dependent mean field pressure shift and broadening of Rydberg lines in gaseous environments and more recently in the context of Rydberg molecule formation in ultracold quantum gases (Greene et al. 2000).

The Fermi pseudopotential is defined is proportional to the scattering length $L_{i}$ of the radiator electron from the $i$-th haze perturber (Fermi 1934), see Fig. 1,

$$
V_{i}\left(\mathbf{r}_{e}, \mathbf{R}_{r}, \mathbf{R}_{i}\right)=\frac{2 \pi \hbar^{2}}{m_{e}} L_{i} \delta\left(\mathbf{R}_{r}+\mathbf{r}_{e}-\mathbf{R}_{i}\right)
$$

where $\mathbf{r}_{\mathbf{e}}$ is the radiator electron coordinate, $L_{i}$ is the scattering length of the electron of mass $m_{e}$ colliding with the haze atom/molecule at $\mathbf{R}_{i}, \hbar$ is the reduced Planck constant, and $\delta()$ is the Dirac delta-function. The chemical composition 


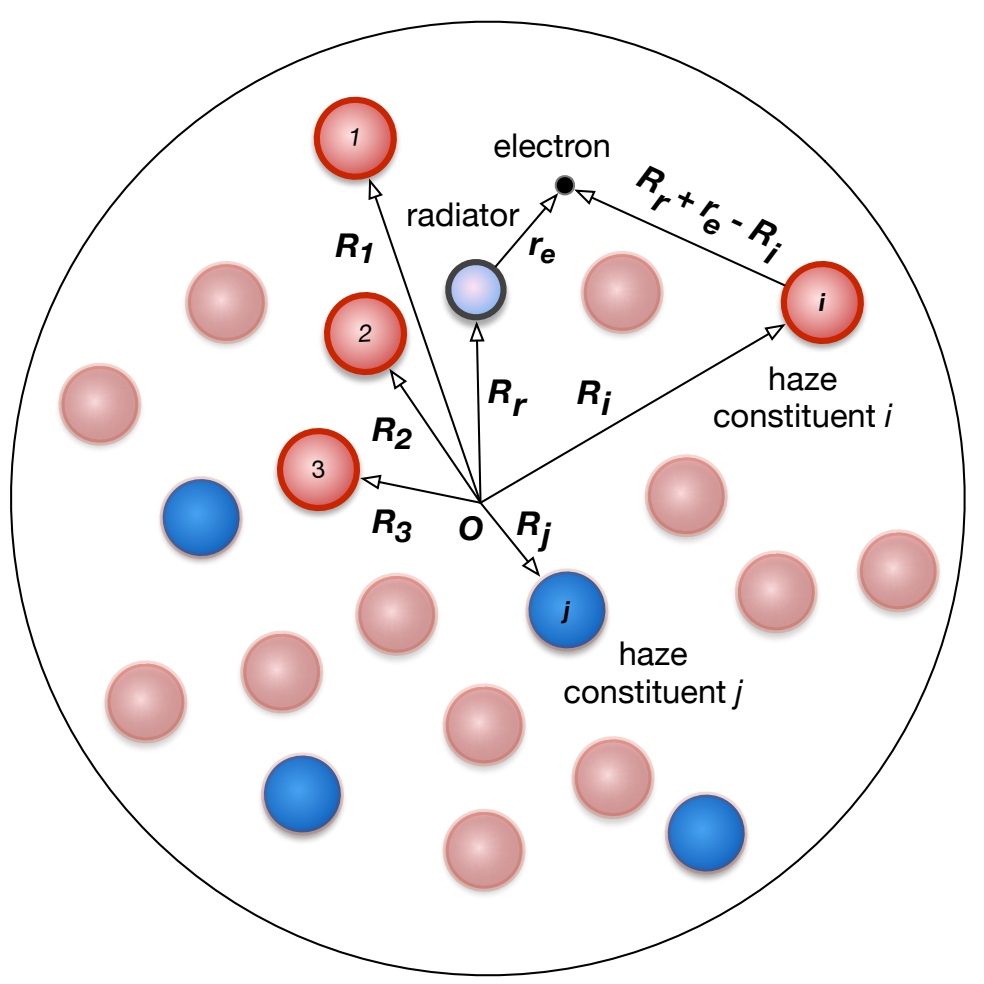

Figure 1. The active radiator electron, $\mathbf{r}_{\mathbf{e}}$, interacts with the radiator core, $\mathbf{R}_{\mathbf{r}}$, and with all other atoms within the haze at positions $\mathbf{R}_{\mathbf{i}}$. Haze constituents of different chemical compositions are denoted with red and blue spheres.

of haze particles is represented by a set of individual scattering lengths $\left\{L_{i}\right\}$. The scattering length is obtained from the zero-energy limit of the scattering phase shift. Presence of perturbers in the vicinity of radiator modifies the radiator wave function and energy spectra that finally leads to changes in energy and rates of emitted/absorbed photons.

Models of spectral shift and broadening (Allard \& Kielkopf 1982; Szudy \& Baylis 1996) describe the influence of collisional or quasi-static interaction potentials on emission and absorption spectra. In the quasi-static approach, the line shift $\Delta \omega=\omega-\omega_{0}$ induced in binary interaction is equal to the difference of radiator electron energies as a function of the perturber-radiator distance, i.e. $\hbar \Delta \omega=\Delta V(R)$, where $\Delta V(R)$ is the difference of the ground and excited BornOppenheimer potential energies, e.g. Sec. 2, induced by the perturber. The inverted equation $R_{C}=R(\Delta \omega)$ yields the location of Condon points, resulting in specific frequency shifts $\Delta \omega$. For the simplest case of cold and dilute gas of uniformly distributed perturbers, the intensity of the spectral line is proportional to the probability to find a perturber at distances between $R$ and $R+d R$, i. e. $I(\Delta \omega)=4 \pi \rho R^{2}(\Delta \omega)\left|\frac{d R(\Delta \omega)}{d(\Delta \omega)}\right|$, with $\rho$ the perturber number density (Allard $\&$ Kielkopf 1982). Haze particles and aggregates may be considered as an environment of slow perturbers with a large and essentially non-uniform perturber number density $\rho(R)$.

The mean-field model in this work, is also applicable to calculations of collisional shift and broadening inside haze aggregate particles with high levels of porosity $(\sim 90 \%)$. Collisional and mean field mechanisms dominate in different regions of emission and absorption spectra. Interaction between haze particles and radiator can provide significant shifts of atomic and molecular lines, in extreme cases up to $10^{4} \mathrm{~cm}^{-1}$ (Jacquet et al. 2011) depending on the radiator position.

Analytical formulas for the shifted electron energies $\epsilon_{i, f}\left(\mathbf{R}_{r}\right)$ of a radiator in the ground (initial) and excited (final) states obtained from the leading term of the perturbation theory:

$$
\epsilon_{i, f}\left(\mathbf{R}_{r}\right)=\epsilon_{i, f}^{0}+\left\langle\Psi_{i, f}\left(\mathbf{r}_{e}\right)\left|V_{i, f}\right| \Psi_{i, f}\left(\mathbf{r}_{e}\right)\right\rangle=\epsilon_{i, f}^{0}+\frac{2 \pi \hbar^{2}}{m_{e}} \sum_{i=1}^{N} L_{i}\left|\Psi_{i, f}\left(\mathbf{r}_{e}=\mathbf{R}_{i}-\mathbf{R}_{r}\right)\right|^{2}
$$

where $\Psi_{i}$ and $\Psi_{f}$ are wave functions of the ground and excited states of free (unperturbed) radiator atoms and $\epsilon_{i, f}^{0}$ are related electronic energies. For systems with high densities of perturbers, such as haze ice, dust or liquid droplets, 
the perturbation can be strong and radiator electronic wave functions need be renormalized according to the mean field induced by the perturbers (Demkov \& Ostrovskii 1988). For haze particles and aggregates with a large number of equivalent atoms or molecules $N$, the summation in Eq. 3 can be replaced with integration over the volume of the haze aggregate with a specific volume density $\rho(\mathbf{R})$. For a collection of $N$ identical perturbers, the Born-Oppenheimer electronic energies of the radiator $\epsilon_{i, f}$ for initial $i$ - and final $f$-states are calculated as:

$$
\epsilon_{i, f}\left(\mathbf{R}_{r}\right)=\epsilon_{i, f}^{0}+V_{i, f}\left(\mathbf{R}_{r}\right)=\epsilon_{i, f}^{0}+\frac{2 \pi \hbar^{2}}{m_{e}} L_{i} N \int d \mathbf{r}_{e} \rho\left(\mathbf{r}_{e}+\mathbf{R}_{r}\right)\left|\Psi_{i, f}\left(\mathbf{r}_{e}\right)\right|^{2}
$$

where $\rho\left(\mathbf{R}_{p}\right)$ is the unit normalized spatial distribution function in the haze droplet at $\mathbf{R}_{\mathbf{p}}=\mathbf{r}_{\mathbf{e}}+\mathbf{R}_{\mathbf{r}}$. The mean-field potential $V_{i, f}\left(\mathbf{R}_{r}\right)$ induced by the haze particles depends on the symmetry of electronic states and symmetry of the perturber distribution function $\rho\left(\mathbf{R}_{p}\right)$. This potential can be a function of the radiator coordinate $\mathbf{R}_{r}$. The spectral intensity $I(\Delta \omega)$ is expressed via mean-filed energy shifts:

$$
I(\Delta \omega)=\int d^{3} R_{r} p\left(\mathbf{R}_{r}\right) \delta\left(\Delta \omega-\left[V_{f}\left(\mathbf{R}_{r}\right)-V_{i}\left(\mathbf{R}_{r}\right)\right] / \hbar\right)
$$

where $p\left(\mathbf{R}_{r}\right)$ is the unit normalized distribution function of radiators inside and outside haze droplet. The replacement of $p\left(\mathbf{R}_{r}\right)$ with the radiator partition function can provide a temperature dependence of the radiator spatial distribution. Angular anisotropy of the mean-field potentials $V_{i, f}\left(\mathbf{R}_{r}\right)$ plays an important role in integration of the $\delta$-function in Eq. 5. For spherically symmetric Born-Oppenheimer energy splitting $\Delta V\left(R_{r}\right)=V_{f}\left(\mathbf{R}_{r}\right)-V_{i}\left(\mathbf{R}_{r}\right)$, the expression for $I(\Delta \omega)$ formally reduces to the known approximation (Allard \& Kielkopf 1982).

\subsubsection{Shift and broadening of spectral line in binary collisions}

The mean-filed spectral shift represents only a part of the total shift and broadening of unperturbed spectral line with frequency $\omega_{0}=\Delta \epsilon_{0}=\epsilon_{i}^{0}-\epsilon_{f}^{0}$. Collisional effects are thermally driven, and hence temperature dependent. In the relatively dense environments, the binary collision between the radiator and a nearby perturber happens in the presence of mean-filed shifts induced by large number of other slowly moving perturbers. The temperature-dependent binary collision broadening and shift rates are, respectively $\rho\left(\mathbf{R}_{r}\right) \gamma(T)$, and $\rho\left(\mathbf{R}_{r}\right) \sigma(T)$ (Allard \& Kielkopf 1982; Vrinceanu et al. 2004).

The overall line shape is obtained upon integrating the localized contributions over the whole volume, assuming that the radiator is found at position $\mathbf{R}_{r}$ with the spherically symmetric probability density $p\left(\mathbf{R}_{r}\right)$ :

$$
I(\omega)=\frac{1}{\pi} \int_{0}^{\infty} \frac{\rho\left(\mathbf{R}_{r}\right) \gamma}{\left[\omega-\left(\Delta \epsilon_{0}+V_{f}\left(\mathbf{R}_{r}\right)-V_{i}\left(\mathbf{R}_{r}\right)\right)-\rho\left(\mathbf{R}_{r}\right) \sigma\right]^{2}+\left[\rho\left(\mathbf{R}_{r}\right) \gamma\right]^{2}} p\left(\mathbf{R}_{r}\right) d^{3} R_{r}
$$

In the above, the first term in the denominator describes the shift of the resonance frequency $\omega_{0}$, and the second term the broadening.

We expect a strong transformation of the emission spectra when the radiator is localized near the surface or inside haze aggregate. In such cases, the strong mean-field interaction creates a broad emission/absorption spectra, significantly shifted from the unperturbed line at $\omega_{0}$. The spectral regions with the photon frequencies near the unperturbed spectral lines are mostly formed by collisional interactions with shifted and broadened Lorentzian profiles (Vrinceanu et al. 2004; Szudy \& Baylis 1996), and become temperature dependent.

The rich experimental and theoretical database on collisional transformation of emission and absorption spectra are available for different atomic and molecular radiators in a gas environment (Szasz 1985; Vrinceanu et al. 2004), where physics of shift and broadening of radiation spectra near the radiator spectral core is described by binary collisions between radiators and gas particles. Analysis of spectral line shift and broadening provides valuable information on parameters of atmospheric gases and haze particles. Parameters of the collisional and mean-field mechanisms of the shift and broadening for different spectral lines can yield unique vistas on physical characteristics of haze particle size, shape and material.

\section{ILLUSTRATIVE RESULTS}

We demonstrate the utility of the current theoretical framework for occultation of expolanetary atmospheres, using sodium atom as the resonant radiator and argon as the haze constituent. The electron scattering length for argon 


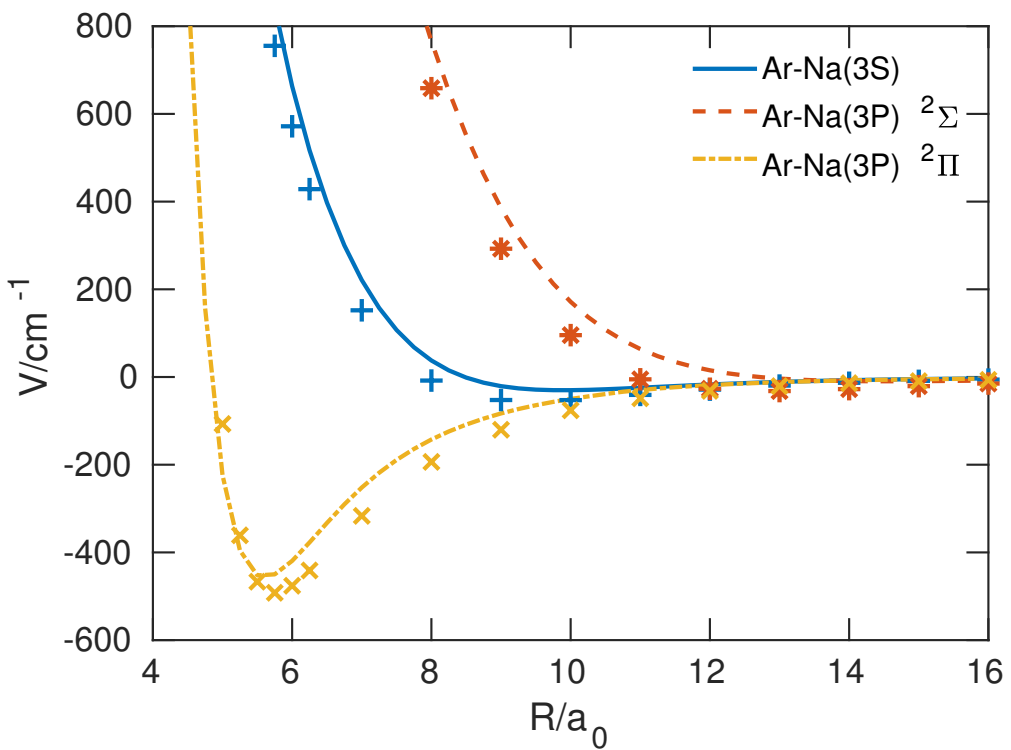

Figure 2. Potential energy curves for $\mathrm{Ar}-\mathrm{Na}(3 \mathrm{~S}, 3 \mathrm{P})$. The $\mathrm{Na} \mathrm{D}$ line energy is subtracted. Lines correspond to the potential energy curves calculated in this work, and symbols correspond to calculated potential energy values (Saxon et al. 1977).

has been measured from low-energy drift velocities of electrons in an H-Ar mixture, to be $L_{A r}=-1.46 a_{0}$, (Petrovic et al. 1995), where $a_{0}$ is the Bohr radius. Admittedly, the haze constituency will more likely be in the form of carbonbearing, water, and other complex molecules, but for purposes of computational efficiency, interacting sodium atoms with rare-gas atomic samples offers a convenient way to calculate many-body mean-field aspect of this theory. Below, first we describe how we calculate the binary interaction in Na-Ar.

\subsection{Binary Potential Energy Curves}

We employ effective core potentials (ECPs), developed by Nicklass et al. (1995), to represent the 10 core electrons of both argon and sodium. The sodium core dipole polarizability is $\alpha=0.9947$ and exponential cutoff parameter $\delta=0.62$, in atomic units, using the core polarization potentials (CPPs) developed by Fuentealba et al. (1982) and implemented in Molpro (Werner \& P. J. Knowles et al. 2012). The one-electron basis set for the valence electrons of the argon perturber is taken from (Nicklass et al. 1995). The sodium radiator is described using an extended one-electron basis set which is defined as follows: The $s, p$, and $d$ orbitals are represented by a set of uncontracted 5 Gaussians with even-tempered exponents between 1.0 and 0.01 atomic units. Two sets of uncontracted Gaussian $f$ orbitals with exponents 0.08 . and 0.008 are also included.

We perform complete active space self-consistent field (CASSCF) calculations to obtain molecular orbitals, which are state-averaged over the $3 \mathrm{~S}$ ground state and $3 \mathrm{P}$ excited states. The active space contains the 9 valence electrons in the argon and sodium $3 s$ and $3 p$ orbitals. Subsequently, we perform multi-reference configuration interaction (MRCI) calculations, which includes single and double excitations from this active space. We include the Pople size-consistency correction, and calculate interaction energies using the counterpoise procedure of (Boys \& Bernardi 1970), to correct for the basis set superposition error. We use a dense grid in the radial coordinate, extending from $R=3$ to $20 a_{0}$ in steps of $0.25 a_{0}$, with additional points at 22, $25 a_{0}$. A final point at $R=50 a_{0}$ is used to subtract any remaining error in size consistency. All calculations are performed using the MolPro suite of ab initio programs (Werner \& P. J. Knowles et al. 2012).

The potential energy curves are shown in Fig. 2. Results obtained in this work are shown as lines. There are two potentials correlating to the $\mathrm{Na}(3 \mathrm{P})$ excited state. The ${ }^{2} \Sigma$ potential governs the interaction if the excited Na valence electron occupies a $3 \mathrm{P}$ orbital oriented parallel to the interatomic axis, whereas the ${ }^{2} \Pi$ potential describes excited states with the $3 \mathrm{P}$ orbital perpendicular to the interatomic axis. The ${ }^{2} \Pi$ excited state potential is significantly more attractive, with a well depth of around $450 \mathrm{~cm}^{-1}$, and becomes repulsive only for much shorter interatomic separations. The ${ }^{2} \Sigma$ excited state, however, has a more repulsive potential than the ground state. The calculated potentials are in good qualitative agreement with the results of Saxon et al. (1977). 

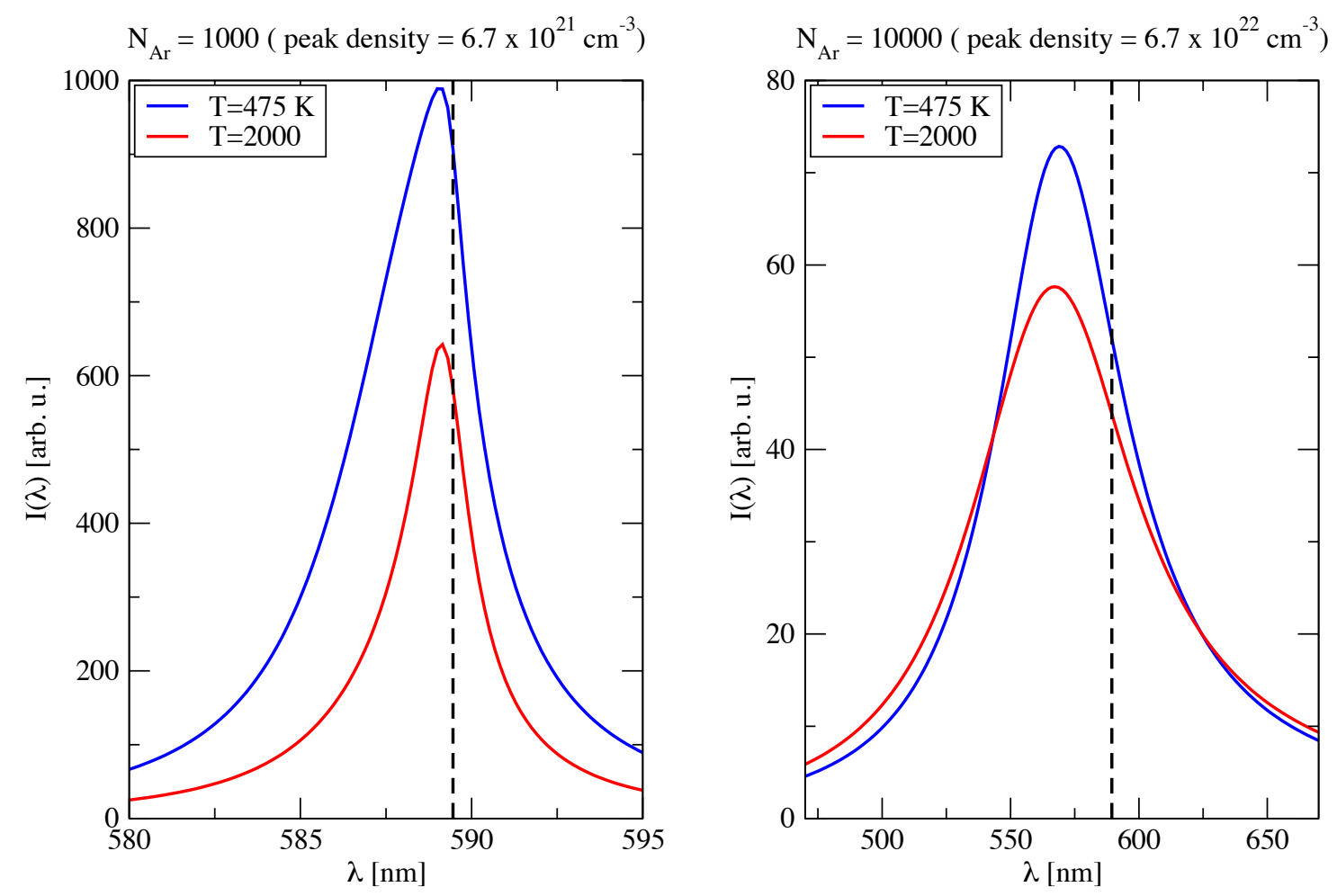

Figure 3. Temperature dependent spectral line profiles of Na D line embedded in an Ar haze of specific peak density. Near the line center, the temperature dependent is the largest due to collisional effects, and away from the center, the lines are no longer Lorentzian as the temperature-insensitive mean field interaction potentials induce asymmetric lineshapes, e.g. Fig. 4.

\subsection{Shift and broadening of Na line in Ar haze}

The argon droplet is modeled by a Gaussian distribution, given by a spherically-symmetric position dependent density $\rho(R)=\left[N_{\mathrm{Ar}} /\left(2 \pi a^{2}\right)^{3 / 2}\right] \exp \left(-R^{2} / 2 a^{2}\right)$, which depends on the droplet size parameter $a$, and the number of $\mathrm{Ar}$ atoms, $N_{\mathrm{Ar}}$. A sodium atom at a distance $R_{r}$ from the center of the droplet has a shifted Lorentz absorption profile as in Eq. 6, where the shape of the line is determined by two contributions: a) mean field quasi-static contribution of neighboring argon atoms that produce the energy shifts $\Delta V\left(R_{r}\right)=V_{3 P}\left(\mathbf{R}_{r}\right)-V_{3 S}\left(\mathbf{R}_{r}\right)$, and b) collisional broadening $\Gamma$ and shift $\Sigma$ rates that are proportional to the local density: $\Gamma=\rho\left(R_{r}\right) \gamma$ and $\Sigma=\rho\left(R_{r}\right) \sigma$. In this model, we use the measured values $\gamma=1.47 \times 10^{-20} \mathrm{~cm}^{-1} / \mathrm{cm}^{-3}$, and $\sigma=0.75 \times 10^{-20} \mathrm{~cm}^{-1} / \mathrm{cm}^{-3}$ at $\mathrm{T}=475 \mathrm{~K}$ (Allard \& Kielkopf 1982). For $\mathrm{T}=2000 \mathrm{~K}$, we multiply the shift and broadening by a factor 1.4; this factor is obtained from a comparison of line broadening in Na-Ar collision from Jongerius et al. (1981).

Fig. 3 illustrates the absorption spectral line shape of the Na D line embedded in a haze environment of specific $\mathrm{Ar}$ density. The two cases considered, with 1000 and $10000 \mathrm{Ar}$ atoms, include haze seed particles of size given by $a=40$ a.u. The probability of finding the sodium atom at some distance $R_{r}$ is given by the Boltzmann distribution, $p\left(R_{r}\right)=\frac{1}{Z} e^{-\epsilon_{3 S}\left(R_{r}\right) / k_{B} T}$, with normalization $Z=\int_{0}^{\infty} e^{-\epsilon_{3 S}\left(R_{r}\right) / k_{B} T}\left(4 \pi R_{r}^{2}\right) d R_{r}, \epsilon_{3 S}\left(R_{r}\right)$ is the mean-field shift in the $3 S$ state, see also Fig. 4 , and $k_{B}$ the Boltzmann constant. In the absence of core broadening due to binary collisions, the spectral line profile will contain a characteristic discontinuity at the $\mathrm{Na}$ line. The line profiles are asymmetric about the radiator line center; this is an expectation of the change in sign of $\Delta \epsilon(R)$, see Fig. 4. In situ simulated laboratory measurements of Titan atmospheric aerosol density confirm that effective number densities of aerosol/haze particle materials are about $10^{22} \mathrm{~cm}^{-3}$ for a methane haze (Hörst \& Tolbert 2013), in accord with our illustrative haze particle densities.

\section{SUMMARY}

In this work, a unified mean field framework for the spectral shift and broadening of an atomic radiator in a dense haze environment has been developed. Both collisional interaction, affecting the line center, and the mean field energy shifts due the presence of other atomic or molecular perturbers affecting the line wings, in hazy environments, are 


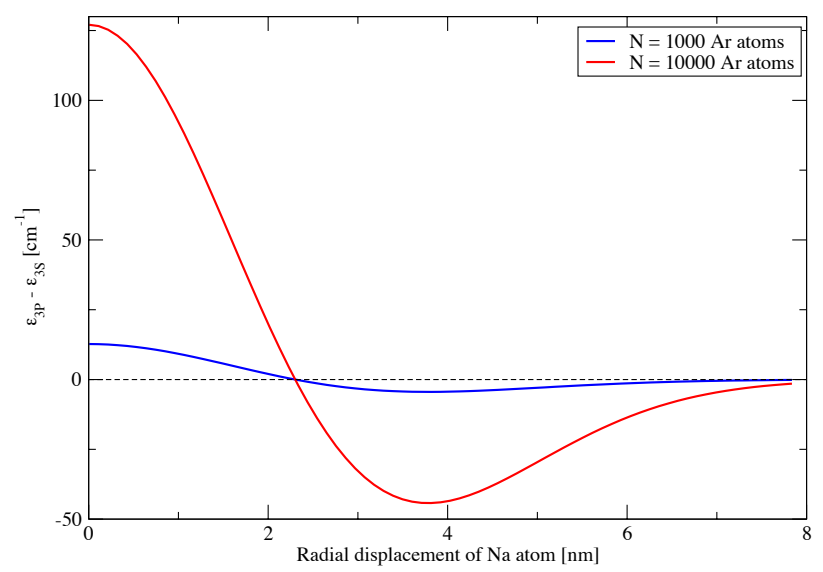

Figure 4. Distortion of the energy difference between levels $\mathrm{Na}(3 \mathrm{~S}, 3 \mathrm{P})$, due to Ar haze mean field, as a function of the position of the $\mathrm{Na}$ atom, for two different numbers of Ar atoms (peak densities $p\left(R_{r}=0\right)$ at $R_{r}=0$ ). The haze size parameter is $a=40$ $\mathrm{a}_{0}(2.12 \mathrm{~nm})$ in both cases, see Sec. 3.2, corresponding to peak densities of Ar atoms, $6.7 \times 10^{21} \mathrm{~cm}^{-3} \mathrm{and}^{6.7 \times 10^{22}} \mathrm{~cm}^{-3}$ for $N=1000$ and $N=10000$, respectively.

accounted for. The collisional contribution depends strongly on temperature and has a Lorentzian form, but the mean field shift is temperature insensitive. The line profiles in hazy environments are asymmetric and significantly broaden because the mean-field generated potential.

The current model has considerable flexibility for extension to different haze environments when chemical composition is mixed, particle densities vary from gaseous to solid phases, and variable porosities. The atomic radiator can be embedded within the haze, or outside and can radiate from high principal quantum numbers, where strong line shift and broadening may inhibit excitations in the first place. The collisional shift and broadening can be quantitatively calculated at various temperatures with accurate quantum mechanical methods in impact approximation (Vrinceanu et al. 2004). The current model can be extended to a description of the radiator embedded into liquid or solid matrix.

\section{ACKNOWLEDGEMENTS}

Z. F. was supported by an ITAMP faculty fellowship from an underrepresented institution. HRS and JFB were supported by an NSF grant to ITAMP. DV was supported by an NSF RISE grant to Texas Southern University. 


\section{REFERENCES}

Allard, N., \& Kielkopf, J. 1982, Rev. Mod. Phys., 54, 1103

Boatz, J., \& Mario, E. 1994, J. Chem. Phys., 101, 3472

Boys, S. F., \& Bernardi, F. 1970, Mol. Phys., 19, 553

Burrows, A., Hubbard, W. B., Lunine, J. I., \& Liebert, J. 2001, Reviews of Modern Physics, 73, 719

Burrows, A., Marley, M. S., \& Sharp, C. M. 2000, ApJ, 531,438

Burrows, A. S. 2014, Nature, 513, 345

Deming, D., \& Seager, S. 2017, ArXiv e-prints, arXiv:1701.00493 [astro-ph.EP]

Demkov, Y., \& Ostrovskii, V. 1988, Zero-Range Potentials and Their Applications in Atomic Physics (Premium Press, NY), 287

ExoEU. 2018, The Extrasolar Planets Encyclopaedia, http://www. exoplanet.eu/

ExoOrg. 2018, Exoplanet Data Explore, http://www . exoplanets.org/

Fermi, E. 1934, Il Nuovo Cimento (1924-1942), 11, 157

Fortney, J. J., Sudarsky, D., Hubeny, I., et al. 2003, ApJ, 589,615

Fuentealba, P., Preuss, H., Stoll, H., \& Szentpály, L. V. 1982, Chem. Phys. Lett., 89, 418

Greenberg, J., \& Li, A. 1999, Space Sc. Rev., 90, 149

Greene, C. H., Dickinson, A. S., \& Sadeghpour, H. R. 2000, Physical Review Letters, 85, 2458

Hörst, S. M., \& Tolbert, M. A. 2013, The Astrophysical Journal Letters, 770, L10

Hörst, S. M., He, C., Lewis, N. K., et al. 2018, ArXiv e-prints, arXiv:1801.06512 [astro-ph.EP]

Jacquet, E., Zanuttini, D., Douady, J., \& et al. 2011, J.Chem.Phys., 135, 174503

Jongerius, M., Bergen, A. V., Hollander, T., \& Alkemade, C. 1981, Journal of Quantitative Spectroscopy and Radiative Transfer, 25, 1

Kreidberg, L., Bean, J. L., Désert, J.-M., et al. 2014, Nature, 505, 69

Lenoble, J., Mishchenko, M. I., \& Herman, H. 2013, In Aerosol Remote Sensing (J. Lenoble et al., Eds.), pp 13-51, 13

Morley, C. V., Fortney, J. J., Marley, M. S., et al. 2015, The Astrophysical Journal, 815, 110
Nicklass, A., Dolg, M., Stoll, H., \& Preuss, H. 1995, J. Chem. Phys., 102, 8942

Ortiz, J. L., Moreno, F., \& Molina, A. 1996, Icarus, 119, 53

Petrovic, Z. L., O’Malley, T. F., \& Crompton, R. W. 1995, Journal of Physics B: Atomic, Molecular and Optical Physics, 28, 3309

Pont, F., Knutson, H., Gilliland, R. L., Moutou, C., \& Charbonneau, D. 2008, MNRAS, 385, 109

Pont, F., Sing, D. K., Gibson, N. P., et al. 2013, MNRAS, 432, 2917

Rannou, P., Hourdin, F., \& McKay, C. 2002, Nature, 418, 853

Saxon, R. P., Olson, R., \& Liu, B. 1977, J. Chem. Phys., 67, 2692

Sciamma-O'Brien, E., Upton, K., \& Salama, F. 2017, Icarus, 289, 214

Seager, S., \& Deming, D. 2010, ARA\&A, 48, 631

Seager, S., \& Sasselov, D. D. 2000, ApJ, 537, 916

Seignovert, B., Rannoua, P., Lavvasa, P., \& et al. 2017, Icarus, 292, 13

Sing, D. K., Désert, J.-M., Fortney, J. J., et al. 2011, A\&A, 527, A73

Spake, J. J., Sing, D. K., Evans, T. M., et al. 2018, Nature, 557,68

Szasz, L. 1985, Pseudopotential theory of atoms and molecules (John Wiley and Sons, Inc., New York)

Szudy, J., \& Baylis, W. 1996, Physics Reports, 266, 127

Tinetti, G., Vidal-Madjar, A., Liang, M.-C., et al. 2007, Nature, 448, 169

Tomasko, M. G., Karkoschka, J., \& Martinek, S. 1986, Icarus, 65, 218

Vrinceanu, D., Kotochigova, S., \& Sadeghpour, H. R. 2004, Phys. Rev. A, 69, 022714

Werner, H.-J., \& P. J. Knowles et al. 2012, MOLPRO: a package of ab initio programs, version 2012.1

West, R. A.and Smith, P. H. 1991, Icarus, 90, 330

Wong, A., Yung, Y. L., \& Friedson, A. J. 2003, Geophys.Res.Lett, 30, 8

Zhang, X., Strobel, D. F., \& Imanaka, H. 2017, Nature, $551,352 \mathrm{EP}$ 